\title{
Compassionate use of a custom 3D-printed sacral implant for revision of failing sacrectomy: case report
}

\author{
Grégoire P. Chatain, MD, and Michael Finn, MD \\ Department of Neurosurgery, University of Colorado School of Medicine, Aurora, Colorado
}

\begin{abstract}
Reconstruction of the spinopelvic continuity after sacral resection for primary sacral tumors remains challenging. Complex anatomical and biomechanical factors of this transition zone may be addressed with the advancement of 3D-printed implants. Here, the authors report on a 67-year-old patient with a sacral chordoma who initially underwent total en bloc sacrectomy followed by standard spinopelvic reconstruction. Pseudarthrosis and instrumentation failure of the lumbosacral junction construct subsequently developed. A custom 3D-printed sacral prosthesis was created using high-resolution CT images. Emergency Food and Drug Administration approval was obtained, and the custom device was implanted as a salvage reconstruction surgery. Made of porous titanium mesh, the custom artificial sacrum was placed in the defect based on the anticipated osteotomic planes and was fixed with a screw-rod system along with a fibular bone strut graft. At the 18-month follow-up, the patient was disease free and walking short distances with assistance. CT revealed excellent bony incorporation into the graft.
\end{abstract}

The use of a custom 3D-printed prosthesis in spinal reconstruction has been rarely reported, and its application in sacral reconstruction and long-term outcome are novel. While the implant was believed to be critical in endowing the region with enough biomechanical stability to promote healing, the procedure was difficult and several key learning points were discovered along the way.

https://thejns.org/doi/abs/10.3171/2020.3.SPINE191497

KEYWORDS spine; sacrectomy; chordoma; prosthesis; 3D printing; sacral

$\mathrm{P}$ RIMARY tumors of the sacrum including chordomas are rare. ${ }^{1}$ Their surgical management remains challenging, as complex anatomical and biomechanical factors of this transition zone have to be taken into account. ${ }^{2}$ With the accumulation of surgical experiences and the advent of more modern surgical techniques, ${ }^{3}$ one-stage total en bloc sacrectomy is becoming frequently used to treat many of these tumors. Wider surgical margins with aggressive resection have shown a remarkable difference in recurrence as compared to that with subtotal excision (51 vs 17.5 months mean disease-free survival) ${ }^{4}$ However, conventional reconstruction of the spinopelvic continuity poses a complex problem, as no standardized surgical treatment has been established. ${ }^{5-10}$ Reestablishing spinopelvic continuity remains a morbid undertaking with a high risk of complications, as it relies on multiple levels of instrumentation and often prolonged bed rest.

3D printing technologies provide the ability to create unparalleled representations of unique patient anatomy and have been used both for surgical planning ${ }^{11}$ and as aids in the construction of 3D-printed implants during sacral reconstruction. ${ }^{12,13}$ With contemporary 3D printing techniques, it is now possible to precisely design and reconstruct bony defects with implants closely tailored to a patient's unique anatomical contours and increase the surface contact between native anatomy and this implant. A porous surface can be given to the implant, which may aide bony integration and result in lower rates of pseudarthrosis. ${ }^{14}$ Custom 3D-printed implants have been used in orthopedics for joint-saving tumor resection and acetabular reconstruction but rarely for sacral reconstrunction. ${ }^{15,16}$ However, long-term functional outcomes of 3D-printed prostheses have seldom been published, and their use remains sporadic.

We present a unique case of the compassionate use of a custom 3D-printed sacral implant for revision of a failing sacrectomy following traditional sacral reconstruction.

\section{Case Report}

History and Examination

A 67-year-old woman presented to our clinic with bi- 

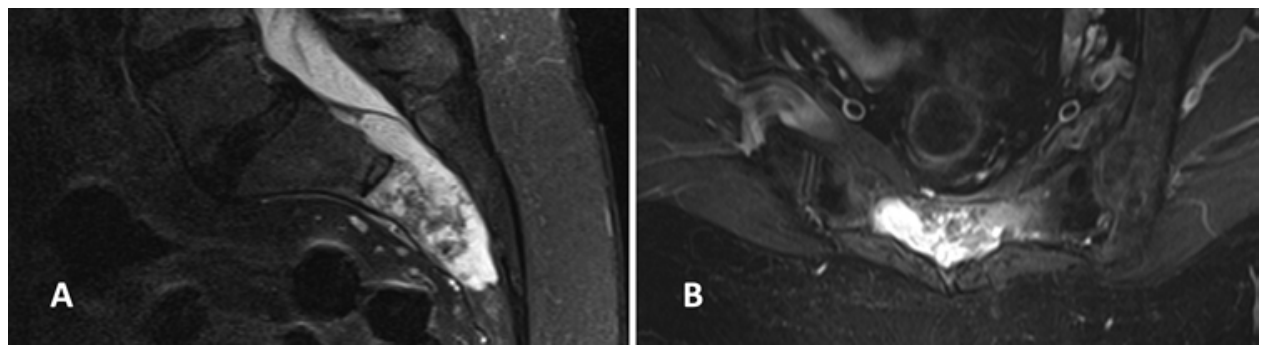

FIG. 1. Preoperative sagittal $(\mathbf{A})$ and axial $(\mathbf{B})$ postcontrast T1-weighted MR images reveal that the mass is centered at the $S 2$ and S3 segments with cortical breakthrough posteriorly and invasion of the epidural space. The mass extends superiorly in the spinal canal along the posterior margin of the S1 vertebral body. The mass is separate from the S1 sacral nerve roots but invades both the left and right S2 sacral foramina.

opsy-proven sacral chordoma symptomatic with bladder and bower dysfunction. Her neurological examination was otherwise nonfocal. Pelvic MRI revealed a $2.9 \times 1.7$ $\times 5.1-\mathrm{cm}$ heterogeneously enhancing mass centered at the S1-3 segments with predominantly posterior extension through the sacral cortex and associated with thecal sac compression (Fig. 1). A treatment plan was decided in a multidisciplinary tumor board consisting of both neurooncologists and radiation oncologists as well as neurosurgeons. After a thorough workup was completed to rule out metastatic disease, the patient elected to pursue surgical intervention divided into two stages. The first stage of the procedure entailed an anterior approach during which the great vessels were dissected off the ventral aspect of the sacrum, and a permanent colostomy was created 2 days later in anticipation of significant bowel and/ or bladder disturbances from her sacrectomy. During the second stage, the patient underwent a posterior total en bloc sacrectomy with negative margins during which the sacral nerve roots were ligated from S1 caudally. This was followed by standard spinopelvic reconstruction. Unfortunately, her recovery was complicated by psoas abscess, infection, deep vein thrombosis, and problems with wound healing requiring multiple wound washouts and revisions with gluteal musculocutaneous rotational and paraspinous
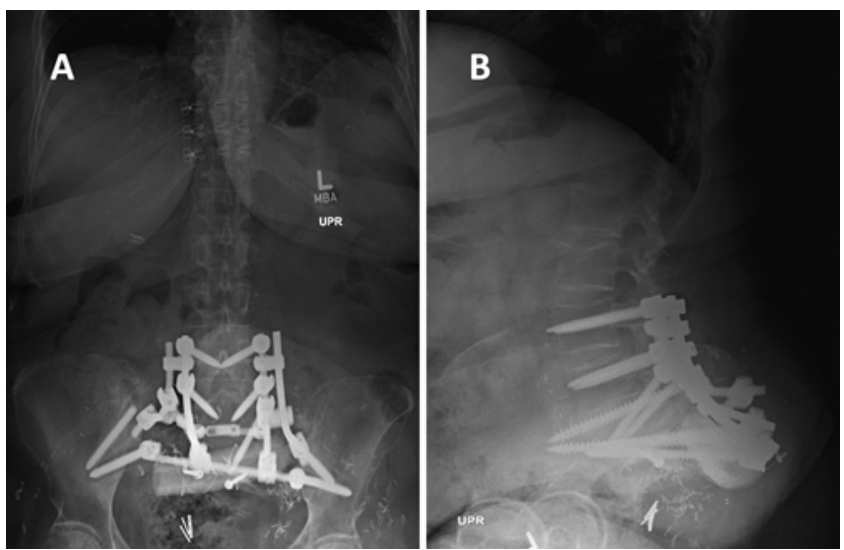

FIG. 2. Lumbar spine radiographs, anteroposterior (A) and sagittal (B) views, demonstrate pseudarthrosis and instrumentation failure of the lumbosacral junction construct. Perimetallic lucency around bilateral iliac screws and fracture of the more superior right iliac screw are visible. muscle flaps. Residual bilateral distal weakness, mainly in plantarflexion, was noted at the clinic follow-up; however, she was able to stand on her own and take a few steps with some assistance. One year postoperatively, she reported increasingly disabling low-back pain and a rising narcotic requirement. Imaging revealed clear pseudarthrosis and instrumentation failure of the lumbosacral junction construct (Fig. 2). As there were no signs of oncological recurrence, a customized 3D-printed sacral prosthesis was prepared for a salvage reconstruction surgery.

\section{D Implant Design and Manufacture}

3D Systems provided Virtual Surgical Planning (VSP) and collaborated with $\mathrm{K} 2 \mathrm{M}$ for the manufacture of the prosthesis. Based on the patient's CT scan, a 3D-reconstructed model was used to define the anatomical structure of the sacral defect and the prospective osteotomy planes for better bony apposition to increase biomechanical stability. 3D Systems developed a virtual surgical plan for the operation so that the 3D-printed sacral implant was designed to physically support the L5 level and bilateral iliac crests through close apposition of surfaces (Supplemental Data). The final prosthesis was created using 3D System's 3D printing capabilities and K2M's Lamellar 3D Titanium Technology (Fig. 3A). A porous structure utilizing 500- $\mu \mathrm{m}$ pore channels alongside a surface roughness of about 3-5 $\mu \mathrm{m}$ was created along all interfaces apposing native bone to promote bony ingrowth, as demonstrated in previous studies. ${ }^{14,17,18}$ The advantage conferred by porosity is its ability to provide biological anchorage for adjacent bone tissue to promote the ingrowth of mineralized tissue into pore spaces. The mechanical stimulation differential along the porous scaffolding of the bone-implant interface is believed to induce migration, differentiation, and proliferation of osteogenic cells..$^{17,18}$ Several plastic implants were initially created to confirm the ease of insertion of the prosthesis, which concomitantly allowed the creation of screw trajectory holes. Once our mock-up was finalized, emergency Food and Drug Administration (FDA) approval was obtained via the emergency and compassionate use of unapproved devices. Given the complexity of implant concept modeling and the FDA approval process, the length of time from surgeon request to implantation was 4 months. The lead time from surgeon approval of the final design to surgical implantation was 6 weeks (Fig. 4). 

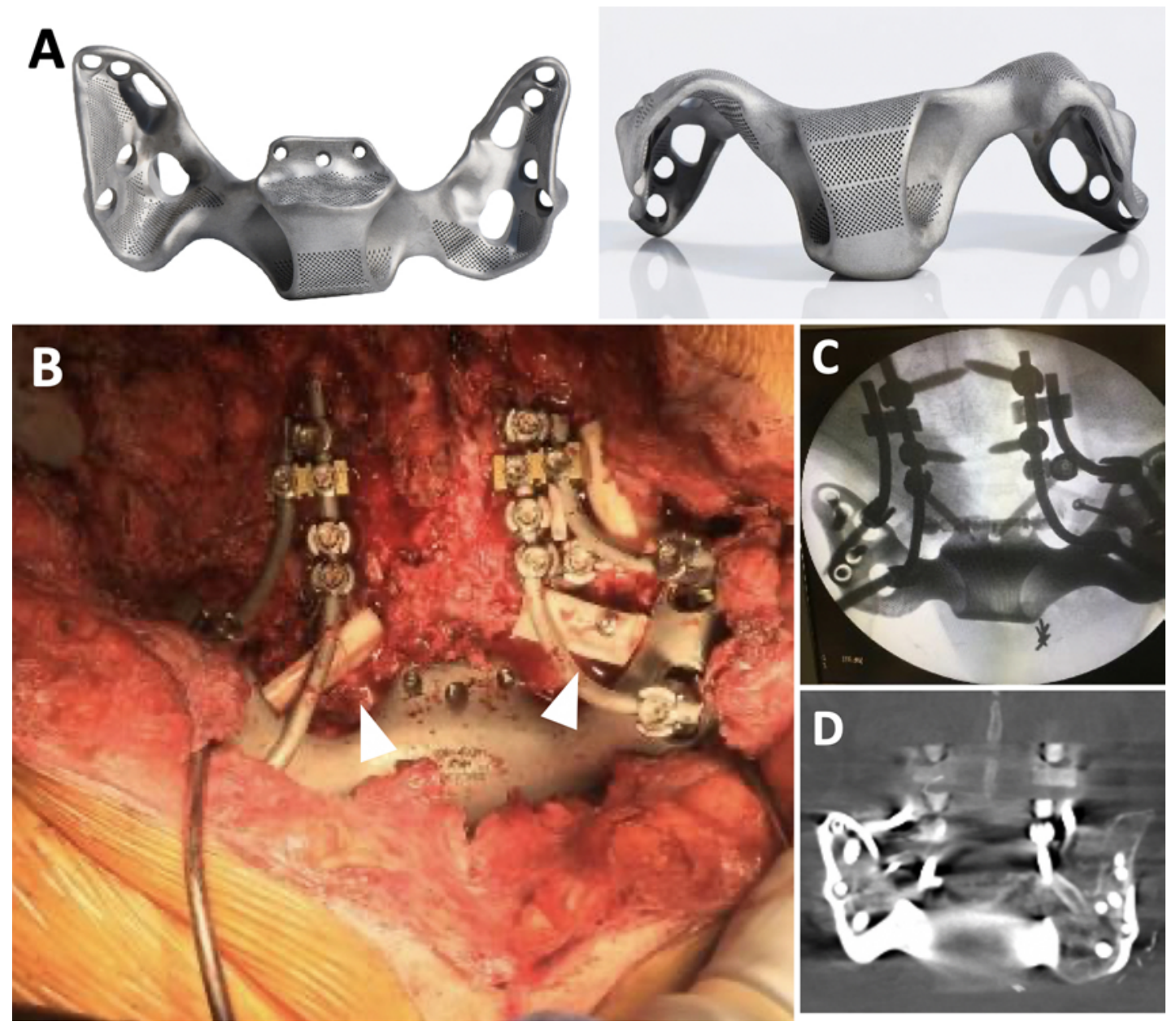

FIG. 3. Based on the patient's CT scan, a 3D reconstruction model was used to define the anatomical structure of the sacral defect and the osteotomy planes for better bony apposition to increase biomechanical stability. The prosthesis was manufactured using a $3 \mathrm{D}$ printing technique and was produced from titanium alloy. A porous structure was created along all interfaces apposing native bone to promote bony ingrowth. Screw trajectory holes were also devised (A). Intraoperative imaging of the implant is shown from the surgeon's view (B). Note the structural fibular strut grafts secured to bridge the lumbar spine to the pelvis (arrowheads, B) and the intraoperative radiograph from the $\mathrm{C}$-arm confirming appropriate placement of hardware (C). At the 1-year follow-up, a coronal CT scan shows adequate bone growth up to the implant surface, whereas no evidence of lucency between implant and bone is visible (D). Note that this device has not been approved or cleared for commercial use or distribution by the FDA. Copyright Stryker. Published with permission.

\section{Surgical Procedure}

Prior to surgery, there were no signs of recurrence or ongoing infection; however, thorough consent was obtained, and the increased risk of hardware infection and theoretical risks of potentially neoplastic growth secondary to the use of bone morphogenetic protein (BMP) were discussed with the patient. At surgery, she was placed prone, and most of the distal hardware was removed. Screws from the L3 to L5 levels were left in place, as the fusion mass was found to be robust on inspection. Unfortunately, given settling of the spine in relation to the pelvis, the implant did not simply slide into the patient's anatomy. The posterior aspect of the iliac crests had to be milled approximately a quarter of an inch bilaterally to line up with the horizontal edge of the implant. Additionally, the lumbar spine and pelvis had to be distracted to a small extent to accommodate the implant surface. The prosthesis was subsequently secured with screws through the im- plant into the iliac crests and L5 bone. Iliac bolts through the implant into the ilium were placed bilaterally under stereotactic navigation. Additionally, the implant provided windows, which constrained screw placement to a degree, assisting implantation down a unique tract not affected by prior screws and pseudarthrotic bone erosion. A rod construct connecting the lumbar to iliac screws was created, and a series of cross-connectors bolstering this construct were used (Figs. 3 and 5). Fibular strut bone grafts from the transverse processes of L3 to L5 to the iliac wings were placed along with BMP and morselized bone graft (Fig. 3B). The wound was closed with the help of plastic surgery. The total procedure time was 8 hours, and the estimated blood loss was $1500 \mathrm{ml}$.

\section{Postoperative Course}

Our patient was discharged on postoperative day 7 and was placed on 3 months of bed rest. At her 1-year 


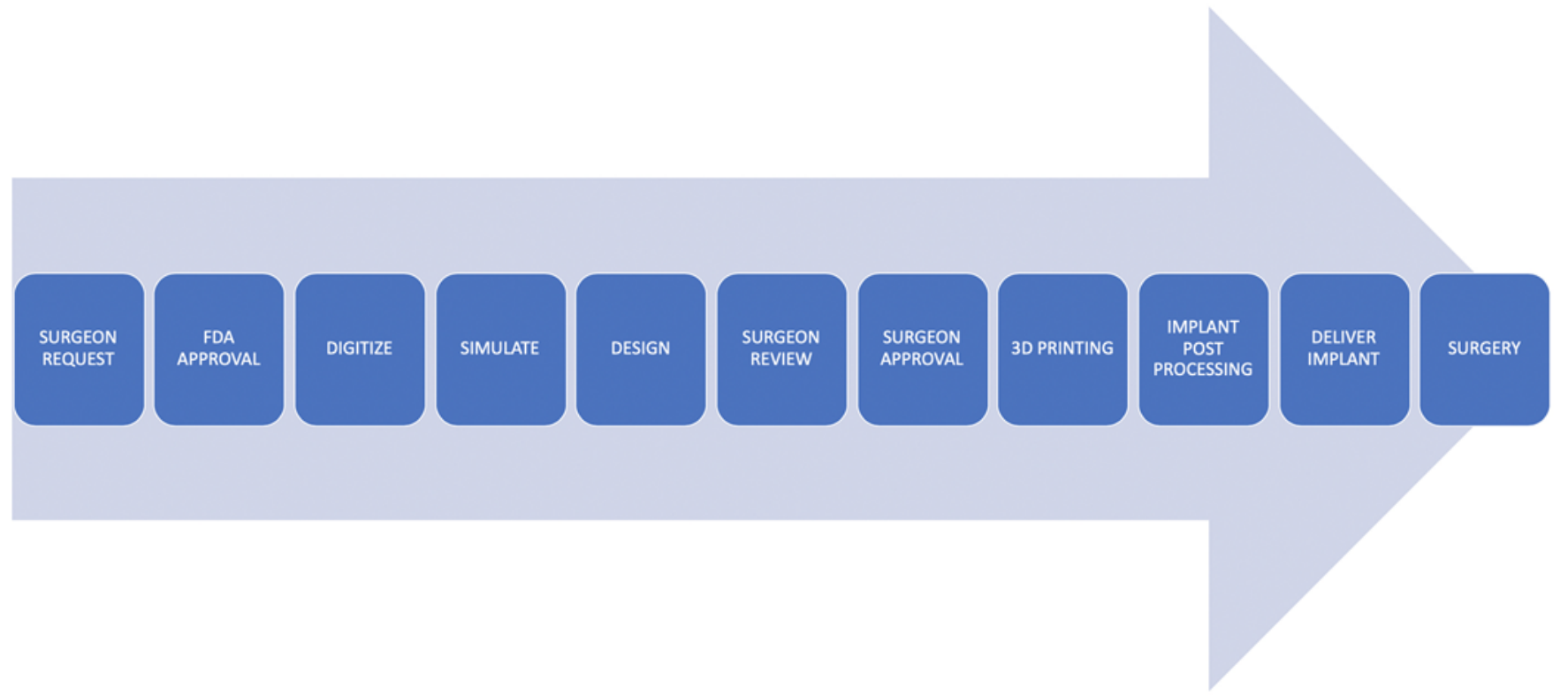

FIG. 4. Workflow diagram for the 3D implant design process. Sequential steps from surgeon request to surgery are broken down. Given the complexity of implant concept modeling and the FDA approval process, the length of time from surgeon request to implantation was 4 months. The lead time from surgeon approval of the final design to surgical implantation was 6 weeks. Copyright Stryker. Published with permission. Figure is available in color online only.

follow-up, she was walking short distances with the aid of a walker. CT revealed bony incorporation into the graft and bridging of the graft directly from the lumbar spine to the pelvis (Figs. 3D and 5). At the 18-month mark, local- ized pain was noted in the left sacroiliac area and believed to be consistent with hardware prominence. Our patient remains disease free and ambulatory at 2 years with radiographs confirming solidification of the bone graft.
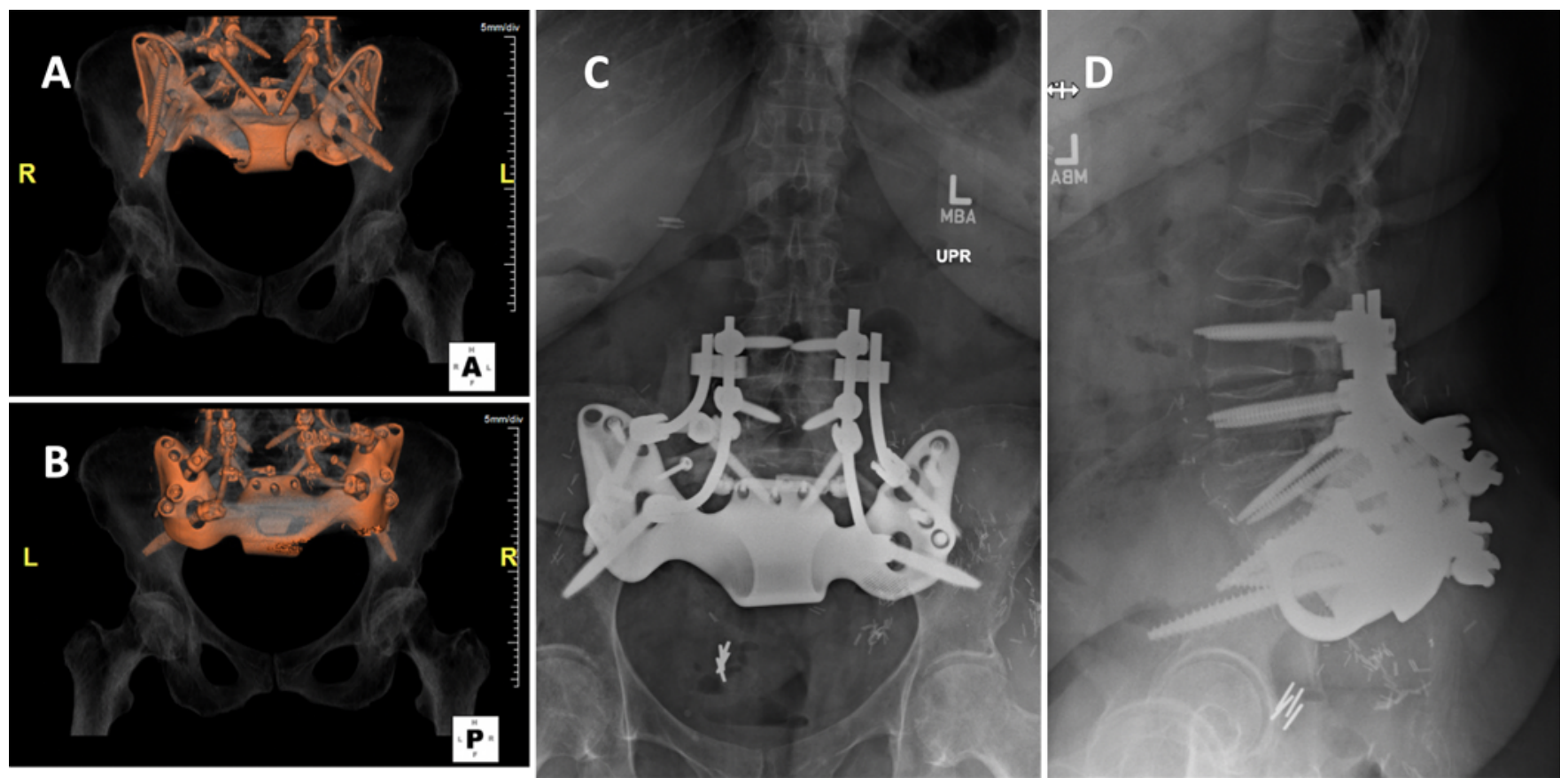

FIG. 5. Interval revision of lumbosacral hardware and reconstruction of spinopelvic continuity with the new 3D-printed sacral prosthesis. As seen on CT reconstruction sequences (A and B) and radiographs, anteroposterior (C) and sagittal (D) views, the custom implant consists of an artificial sacrum with edges abutting the caudal edge of the $L 5$ vertebral body and the iliac wings. The implant was placed on the defect based on the anticipated osteotomic planes and was fixed with a screw-rod system along with fibular bone strut graft from $\mathrm{L} 3$ to the iliac wings. 


\section{Discussion}

Sacral chordomas are difficult lesions to treat surgically mainly because of the complex anatomy of the spinopelvic transition zone and the need for complex reconstruction techniques. Aggressive en bloc excision remains the central tenant in the surgical management, and advancements in techniques have allowed for more efficient procedures with reduced morbidity. ${ }^{1,710,19}$ Despite major advances in surgical approaches, total en bloc resection is only attainable in about $50 \%$ of patients suffering from sacral chordomas and recurrence is high. ${ }^{1,20,21}$

Although sacrectomy techniques have become safer and more efficient, the methods of spinopelvic reconstruction remain suboptimal after decades of effort. $2,7,10,22-28$ Many patients end up with a large dead space and poor soft tissue coverage after surgical excision, often requiring the involvement of plastic surgery and the rotation of a flap to increase the odds of wound healing. Clinical studies examining failure rates and biomechanical studies have shown that conventional reconstruction methods should include spinal pelvic fixation, posterior pelvic ring fixation, and anterior spinal column support. ${ }^{5,22}$ However, it remains difficult to accomplish these goals satisfactorily with conventional techniques given the anatomical and load-bearing characteristics of the lumbopelvic junction.

3D printing is a rapidly evolving technology, which has recently been applied to the creation of surgical implants. It has been successfully applied in both the planning for ${ }^{11}$ and the reconstruction of upper cervical spine ${ }^{29}$ and pelvic ${ }^{12,13,30}$ bony defects as well as orthopedic applications. ${ }^{15,16}$ While these custom implants should provide greater anatomical stability, and therefore improved success rates, there is a paucity of formal biomechanical testing in the literature, and the long-term functional and clinical outcomes of custom 3D-printed implants remain largely unknown. To our knowledge, this is the first report of a custom 3D-printed sacral implant procedure used as a salvage operation for a failed lumbosacral reconstruction.

In this present case, we used a 3D-printed prosthesis that was designed to fit the anatomical structure of the sacral defect and maximize bony apposition, thereby creating sufficient biomechanical stability in the region to allow for bony fusion. The titanium alloy prosthesis also used a porous structure to promote bony ingrowth. ${ }^{14,17,18}$ As demonstrated at the long-term follow-up, these features likely assisted in the successful healing of the region in this patient.

Surgically implanting the device was a significant task. One of the bigger challenges faced was progressive settling of the spine from the time of the measurement CT to device manufacture. Although emergency FDA approval was obtained, the lead time to implantation was 6 weeks, and this was enough to allow a degree of settling to complicate implantation. Intraoperative modification of the implant is limited, and implantation required greater than anticipated milling of native bone and distraction of the lumbosacral spine. Although such implants may be considered for use in an index procedure in the future, it is currently cost-prohibitive as the prosthesis had to be custom-made with no off-shelf equivalent. Moreover, such an implant may be suboptimal when follow-up imaging is needed secondary to extensive metal artifact. Prior to its widespread use, more experience is required, FDA hurdles must be cleared, and a shorter lead time for custom-made sacral implants should be sought.

\section{Conclusions}

The use of a custom 3D-printed prosthesis in spinal reconstruction has been rarely reported, and its use in sacral reconstruction as well as its long-term outcome is novel. We believe that the creation of a custom 3D-printed implant is a viable option for use in a salvage procedure for patients in whom conventional lumbosacral reconstructions have failed, and we have shown it to be successful in this single case report. As demonstrated in our case, the lead time from device manufacture to implantation was problematic; therefore, efforts must be made to minimize this.

\section{References}

1. Walcott BP, Nahed BV, Mohyeldin A, et al. Chordoma: current concepts, management, and future directions. Lancet Oncol. 2012;13(2):e69-e76.

2. Zhang H-Y, Thongtrangan I, Balabhadra RSV, et al. Surgical techniques for total sacrectomy and spinopelvic reconstruction. Neurosurg Focus. 2003;15(2):E5.

3. Stener B, Gunterberg B. High amputation of the sacrum for extirpation of tumors. Principles and technique. Spine (Phila Pa 1976). 1978;3(4):351-366.

4. Hsieh PC, Xu R, Sciubba DM, et al. Long-term clinical outcomes following en bloc resections for sacral chordomas and chondrosarcomas: a series of twenty consecutive patients. Spine (Phila Pa 1976). 2009;34(20):2233-2239.

5. Bederman SS, Shah KN, Hassan JM, et al. Surgical techniques for spinopelvic reconstruction following total sacrectomy: a systematic review. Eur Spine J. 2014;23(2):305-319.

6. Gallia GL, Haque R, Garonzik I, et al. Spinal pelvic reconstruction after total sacrectomy for en bloc resection of a giant sacral chordoma. Technical note. J Neurosurg Spine. 2005;3(6):501-506.

7. Guo W, Tang X, Zang J, Ji T. One-stage total en bloc sacrectomy: a novel technique and report of 9 cases. Spine (Phila Pa 1976). 2013;38(10):E626-E631.

8. Nishizawa K, Mori K, Saruhashi Y, et al. Long-term clinical outcome of sacral chondrosarcoma treated by total en bloc sacrectomy and reconstruction of lumbosacral and pelvic ring using intraoperative extracorporeal irradiated autologous tumor-bearing sacrum: a case report with 10 years follow-up. Spine J. 2014;14(5):e1-e8.

9. Wuisman P, Lieshout O, Sugihara S, van Dijk M. Total sacrectomy and reconstruction: oncologic and functional outcome. Clin Orthop Relat Res. 2000;(381):192-203.

10. Zang J, Guo W, Yang R, et al. Is total en bloc sacrectomy using a posterior-only approach feasible and safe for patients with malignant sacral tumors? J Neurosurg Spine. 2015;22(6):563-570.

11. Ahmed AK, Pennington Z, Molina CA, et al. Multidisciplinary surgical planning for en bloc resection of malignant primary cervical spine tumors involving 3D-printed models and neoadjuvant therapies: report of 2 cases. J Neurosurg Spine. 2019;30(4):424-431.

12. Kim D, Lim J-Y, Shim K-W, et al. Sacral reconstruction with a 3D-printed implant after hemisacrectomy in a patient with sacral osteosarcoma: 1-year follow-up result. Yonsei Med J. 2017;58(2):453-457.

13. Wei R, Guo W, Ji T, et al. One-step reconstruction with a 3D- 
printed, custom-made prosthesis after total en bloc sacrectomy: a technical note. Eur Spine J. 2017;26(7):1902-1909.

14. Shah FA, Snis A, Matic A, et al. 3D printed Ti6Al4V implant surface promotes bone maturation and retains a higher density of less aged osteocytes at the bone-implant interface. Acta Biomater. 2016;30:357-367.

15. Malik HH, Darwood ARJ, Shaunak S, et al. Three-dimensional printing in surgery: a review of current surgical applications. J Surg Res. 2015;199(2):512-522.

16. Wong KC. 3D-printed patient-specific applications in orthopedics. Orthop Res Rev. 2016;8:57-66.

17. Palmquist A, Snis A, Emanuelsson L, et al. Long-term biocompatibility and osseointegration of electron beam melted, free-form-fabricated solid and porous titanium alloy: experimental studies in sheep. J Biomater Appl. 2013;27(8):10031016.

18. Vasconcellos LM, Leite DO, Oliveira FN, et al. Evaluation of bone ingrowth into porous titanium implant: histomorphometric analysis in rabbits. Braz Oral Res. 2010;24(4):399405.

19. Li D, Guo W, Tang X, et al. Surgical classification of different types of en bloc resection for primary malignant sacral tumors. Eur Spine J. 2011;20(12):2275-2281.

20. Fuchs B, Dickey ID, Yaszemski MJ, et al. Operative management of sacral chordoma. J Bone Joint Surg Am. 2005;87(10):2211-2216.

21. Osaka S, Kodoh O, Sugita H, et al. Clinical significance of a wide excision policy for sacrococcygeal chordoma. J Cancer Res Clin Oncol. 2006;132(4):213-218.

22. Clark AJ, Tang JA, Leasure JM, et al. Gait-simulating fatigue loading analysis and sagittal alignment failure of spinal pelvic reconstruction after total sacrectomy: comparison of 3 techniques. J Neurosurg Spine. 2014;20(4):364-370.

23. Dickey ID, Hugate RR Jr, Fuchs B, et al. Reconstruction after total sacrectomy: early experience with a new surgical technique. Clin Orthop Relat Res. 2005;438:42-50.

24. Le VH, Heckmann N, Jain N, et al. Biomechanical evaluation of supplemental percutaneous lumbo-sacro-iliac screws for spinopelvic fixation following total sacrectomy. J Spinal Disord Tech. 2015;28(4):E181-E185.

25. Lim S-H, Jo D-J, Kim S-M, Lim Y-J. Reconstructive surgery using dual U-shaped rod instrumentation after posterior en bloc sacral hemiresection for metastatic tumor: case report. $J$ Neurosurg Spine. 2015;23(5):630-634.

26. Mindea SA, Chinthakunta S, Moldavsky M, et al. Biomechanical comparison of spinopelvic reconstruction techniques in the setting of total sacrectomy. Spine (Phila Pa 1976). 2012;37(26):E1622-E1627.
27. Shen FH, Harper M, Foster WC, et al. A novel "fourrod technique" for lumbo-pelvic reconstruction: theory and technical considerations. Spine (Phila Pa 1976). 2006;31(12):1395-1401.

28. Yu B-S, Zhuang X-M, Zheng Z-M, et al. Biomechanical advantages of dual over single iliac screws in lumbo-iliac fixation construct. Eur Spine J. 2010;19(7):1121-1128.

29. Xu N, Wei F, Liu X, et al. Reconstruction of the upper cervical spine using a personalized 3D-printed vertebral body in an adolescent with Ewing sarcoma. Spine (Phila Pa 1976). 2016;41(1):E50-E54.

30. Wong KC, Kumta SM, Geel NV, Demol J. One-step reconstruction with a 3D-printed, biomechanically evaluated custom implant after complex pelvic tumor resection. Comput Aided Surg. 2015;20(1):14-23.

\section{Disclosures}

Dr. Finn is a consultant for K2M/Stryker.

\section{Author Contributions}

Conception and design: Chatain. Acquisition of data: Chatain. Analysis and interpretation of data: Chatain. Drafting the article: Chatain. Critically revising the article: Finn, Chatain. Reviewed submitted version of manuscript: Finn, Chatain. Approved the final version of the manuscript on behalf of both authors: Finn. Study supervision: Finn.

\section{Supplemental Information \\ Online-Only Content}

Supplemental material is available with the online version of the article.

Supplemental Data. https://thejns.org/doi/suppl/10.3171/2020. 3.SPINE191497.

\section{Correspondence}

Michael Finn: University of Colorado School of Medicine, Aurora,CO.michael.finn@cuanschutz.edu. 